\title{
Silencing of TCTN1 inhibits proliferation, induces cell cycle arrest and apoptosis in human thyroid cancer
}

\author{
PEIPEI XU ${ }^{1 *}$, XIAOTIAN XIA $^{1 *}$, ZHILI YANG $^{1}$, YUAN TIAN $^{1}$, JIANZHONG DI $^{1,2}$ and MINGGAO GUO ${ }^{1}$ \\ ${ }^{1}$ Department of Surgery, The Sixth People's Hospital of Shanghai, Shanghai Jiaotong University, \\ Shanghai 200233; ${ }^{2}$ Department of Surgery, The People's Hospital of Xigaze, 857000 Tibet, P.R. China
}

Received January 22, 2016; Accepted July 10, 2017

DOI: $10.3892 /$ etm.2017.4940

\begin{abstract}
Tectonic family member 1 (TCTN1) is one of the tectonic family members, and a regulator of the hedgehog signaling pathway, which has been studied in various cancer types, including prostate and pancreatic cancer. However, its function in thyroid cancer has not been well documented. Therefore, the present study investigated the function of TCTN1 in thyroid cancer using a loss-of-function assay. Lentivirus-mediated RNA interference was applied to downregulate TCTN1 in the thyroid cancer cell lines, CAL62 and 8305C. A series of functional properties, including cell viability, colony formation, cell cycle and apoptosis were determined using MTT, colony formation assay and flow cytometry analyses, respectively. The results demonstrated that lentivirus-medicated RNAi could specifically suppress the expression of TCTN1 at the mRNA and protein levels in CAL62, and 8305C cells. Knockdown of TCTN1 inhibited cell growth and proliferation via inducing $\mathrm{S}$ phase arrest, and apoptosis. Mechanistically, the $\mathrm{S}$ phase arrest was accompanied by the upregulation of cyclin dependent kinase 2, cyclin A2 and downregulation of cyclin B1. Knockdown of TCTN1 induced apoptosis through increasing the expression of Bcl2-associated agonist of cell death, cleaved caspase-3 and poly(ADP-ribose)polymerase, and decreasing apoptosis regulator Bcl-2 expression. The current study highlights the essential role of TCTN1 in promoting thyroid cancer cell proliferation, and its knockdown may serve as a potential therapeutic treatment for thyroid cancer.
\end{abstract}

\section{Introduction}

Thyroid cancer, which arises from follicular cells and parafollicular cells, is one of the most rapidly growing endocrine

Correspondence to: Dr Minggao Guo, Department of Surgery, The Sixth People's Hospital of Shanghai, Shanghai Jiaotong University, 600 Yishan Road, Shanghai 200233, P.R. China

E-mail: guominggao203@163.com

*Contributed equally

Key words: thyroid cancer, TCTN1, proliferation, apoptosis malignancies in many countries (1). According to the specific histological features, thyroid cancer can be classified into four types, including papillary carcinoma follicular carcinoma, medullary carcinoma and undifferentiated carcinoma (2). Over the past few decades, diagnostic and therapeutic approaches have greatly improved long-term survival of thyroid cancer, but higher recurrence still is found in most cases (3). Currently, molecular biology has provided possibility to investigate the pathogenesis of thyroid cancer and develop a novel effective therapeutic strategy for thyroid cancer.

Tectonic family member 1 (TCTN1) is one of the tectonic family members, which is related with the development of the cilia of the eukaryotes (4). TCTN1 can regulate the hedgehog signal pathway in mouse (5) and its mutation has influence on the Joubert Syndrome (6-8). Recently, TCTN1 has been demonstrated to be significantly upregulated in various tumors, including glioma (9), gastric cancer (10), prostate cancer (11) and pancreatic cancer (12). These studies further showed that knockdown of TCTN1 could efficiently inhibit cell proliferation in vitro, suggesting it might also be an oncogene in thyroid cancer progression. However, to the best of our knowledge, the potential implication of TCTN1 in the initiation and development has not been well documented.

Therefore, in the present study, we investigate the potential role and molecular mechanism of TCTN1 in thyroid cancer in vitro. We found that the downregulation of TCTN1 suppressed thyroid cancer cell proliferation by inducing cell cycle arrest and apoptosis. These findings might provide some evidences that TCTN1 plays a crucial role in the context of tumorigenesis and proliferation of thyroid cancer.

\section{Materials and methods}

Analysis of oncomine data. In order to determine the expression of TCTN 1 in thyroid cancer, we performed data mining using Oncomine database (www.oncomine.org). An outlier analysis of TCTN 1 was performed in the thyroid cancer tissues.

Cell lines and cell culture. Human thyroid cancer cell lines (CAL62, TT and 8305C) and the packaging cell 293T cell line were obtained from Cell Bank of Chinese Academy of Science (Shanghai, China). Cells were maintained in dulbecco's modified eagle medium (DMEM), supplemented with $10 \%$ fetal 
bovine serum (FBS; Gibco Life Technologies, Beijing, China) at $37^{\circ} \mathrm{C}$ in a humidified atmosphere containing $5 \% \mathrm{CO}_{2}$.

Lentivirus production and cell transfection. The short hairpin oligonucleotides (shTCTN1: 5'-GCTCAGATGCATCAGTT CCTTCTCGAGAAGGAACTGATGCATCTGAGCTTTTT T-3' and shCon: 5'-CAACAAGATGAAGAGCACCAACTC GAGTTGGTGCTCTTCATCTTGTTG-3') were synthesized and cloned into the lentivirus-based vector pGP-L (System Biosciences, Inc., Palo Alto, CA, USA). Lenti virus production was followedas standard protocol by transfecting $293 \mathrm{~T}$ cells with recombinational shRNA vector, together with pVSVG-I and pCMVAR8.92 (Sigma, St. Louis, MO, USA). For cell transfection, thyroid cancer cells were cultured in 6-well plates and transfected with the shTCTN1 or shCon with a multiplicity of infection (MOI) of 60. After $48 \mathrm{~h}$, the transfection efficiency was observed through a fluorescence microscope, and TCTN1 expression was determined using qRT-PCR and western blot assay.

qRT-PCR analysis. Trizol reagent (Invitrogen, Carlsbad, CA, USA) was used to extract RNA and reverse transcription was conducted with the revertra ace qPCR RT kit (TOYOBO Co., Ltd., Osaka, Japan). The PCR primers used were as follows: TCTN1 (forward): 5'-CCTTTGCGTGAA TGTTGTTC-3', TCTN1 (reverse): 5'-AGAGGGACTGGC TGGGTATT-3'; Actin (forward): 5'-GTGGACATCCGCAAA GAC-3', Actin (reverse): 5'-AAAGGGTGTAACGCAACTA-3'. The TCTN1 mRNA expression was evaluated by quantitative PCR on BioRad Connet Real-Time PCR platform (Bio-Rad Laboratories, Hercules, CA, USA). Data were analyzed using the $2^{-\Delta \Delta C t}$ method.

Western blot analysis. The cells were washed twice with ice-cold PBS and lysed in 2X SDS sample buffer [100 mM Tris- $\mathrm{HCl}$ (pH 6.8), $10 \mathrm{mM}$ EDTA, 4\% SDS and 10\% glycine]. Equal amount of proteins $(30 \mu \mathrm{g})$ were loaded and separated by electrophoresis (50 V, $3 \mathrm{~h}$ ) on $10 \%$ SDS-PAGE gels. Western blot was performed according to previous report (13). The primary antibodies used were as follows: Anti-TCTN1, Bad, Bcl-2, Caspase-3, PARP, Cyclin B1, CDK2, Cyclin A2 and Cyclin D1. Horseradish peroxidase-conjugated antibody was used as secondary antibody (1:5,000 dilution, \#SC-2054; Santa Cruz Biotechnology, Inc., Dallas, TX, USA). An anti-GAPDH antibody (1:500,000, 10494-1-AP; ProteinTech group, Chicago, IL, USA) was used as the loading control. In addition, The 9 proteins including Bad, Bcl-2, cleaved caspase-3, PARP, Cyclin B1, CDK2, Cyclin A2, Cyclin D1 and GAPDH were separated on $10 \%$ SDS-PAGE at the same time in different lanes. According to protein molecular weight, SDS-PAGE was cut before transferring electrophoretically onto a PVDF membrane. Bad, CDK2 and cyclin B1 were cut in the same lane at molecular weight 15-25 KD, 25-40 and 40-100 KD. Bcl-2 and cyclin A2 were cut in the same lane at molecular weight 15-35 and 40-70 KD. Cleaved caspase-3 and cyclin D1 were cut at molecular weight 15-25 and 25-40 KD. GAPDH and PARP were cut at molecular weight 15-40 and 55-170 KD.

Cell viability assay. After lentivirus infection, CAL62 and $8305 \mathrm{C}$ cells were reseeded in a 96-well plate, respectively, at a density of 2,500 cell/well. MTT assay was used to determine cell viability at five different time points (day 1,2, 3, 4 and 5). Briefly, each well was added $20 \mu \mathrm{l}$ of MTT (Sigma) solution and incubated for $4 \mathrm{~h}$ at $37^{\circ} \mathrm{C}$. Then $100 \mu \mathrm{l}$ acidic isopropanol (10\% SDS, 5\% isopropanol and $0.01 \mathrm{~mol} / \mathrm{l} \mathrm{HCl})$ was added to stop the reaction. The optical density (OD) value was measured at $595 \mathrm{~nm}$ using a microplate reader (Epoch; BioTek, Winooski, VT, USA). All experiments were performed in triplicates.

Colony formation assay. Colony formation assay was used to evaluate the colony formation ability of thyroid cancer cells after infected with shTCTN1 or shCon according to previous report (14). Briefly, cells (600 cells/well) were reseeded on six-well plates and cultured for 12 days to form natural colonies. Then colonies were fixed using $4 \%$ paraformaldehyde for $5 \mathrm{~min}$ and then stained with $1 \%$ crystal violet for $30 \mathrm{sec}$. The cells were photographed, and the number of colony (more than 50 cells/colony) was counted.

Cell cycle and apoptosis assays. For cell cycle assay, cells were collected, washed with pre-cooling PBS and fixed with cooling $70 \%$ ethanol overnight at $4^{\circ} \mathrm{C}$. After washing twice with pre-cooling PBS, the cells were resuspended and incubated in $500 \mu \mathrm{l}$ PBS containing $50 \mu \mathrm{g} / \mathrm{ml}$ propidium iodide (PI) solution and $100 \mu \mathrm{g} / \mathrm{ml}$ RNase for $30 \mathrm{~min}$ at room temperature.

For apoptosis assay, cells were assessed with Annexin V-APC/AAD staining (eBioscience Inc., San Diego, CA, USA) on a flow cytometer (BD Biosciences, San Diego, CA, USA) following the manufacturer's instruction. Finally, cells were analyzed on a FACS Calibur cytometer (BD Biosciences). Data were analyzed using CellQuest software.

Statistical analysis. All statistical analyses were performed using SPSS version 13.0 software. The differences between groups were compared using the Student's test, and quantitative data are expressed as the means \pm SD of 3 independent experiments. A value of $\mathrm{P}<0.05$ was considered as a statistical significant difference.

\section{Results}

Lentivirus-mediated shTCTN1 efficiently knocks down endogenous expression of TCTN1. Oncomine database supplied us the TCTN1 expression in thyroid cancer tissues. Hence, TCTN 1 expression levels in thyroid cancer tissues were investigated using publicly available Oncomine database (www.oncomine.org). The results showed that no significant changes of TCTN1 expression were observed in thyroid tumor samples. While considering cancer heterogeneity was a potential challenge of proto-oncogene screening, an outlier analysis was performed in thyroid cancer sample for TCTN1 expression and the outlier was set at 75\%. As shown in Fig. 1, the expression of TCTN 1 showed a significantly increased in 7 databases from different studies. These results demonstrated that TCTN 1 was overexpressed in certain thyroid cancer and may be a proto-oncogene in thyroid cancer.

Now that TCTN1 has been demonstrated overexpressed in thyroid cancer tissue, we deduced that a similar phenomenon 
TCTN1 Copy Number in TCGA Thyroid

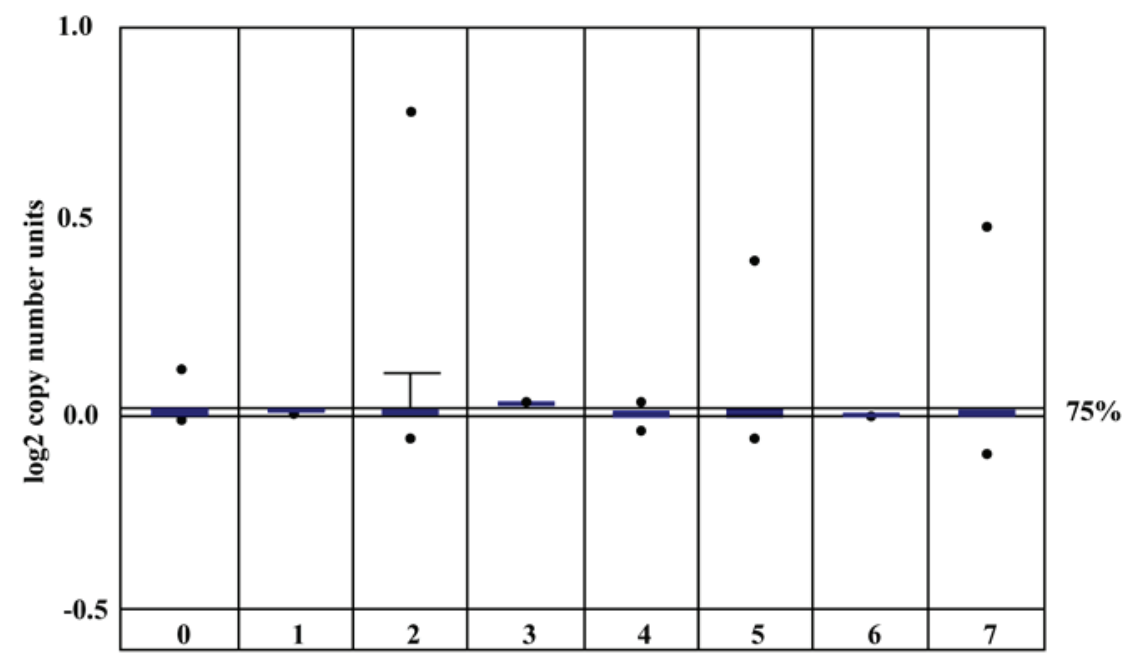

Legend

\section{No value (507) \\ 1. Colimnal cell Variant Thyroid \\ Gland Papollary Carcinoma(1)}

2. Follicular Variant Thyroid

Gland Papillary Carcinoma (95)

3. Oncocytic Variant Thyroid

Gland Papillary Carcinoma (1)
4. Tall Cell Variant Thyroid

Gland Papillary Carcinoma (34)

5. Thyroid Gland Carcinoma (45)

6. Thyroid Gland Diffuse Sclerosing Papillary Carcinoma (2)

7. Thyroid Gland Papillary Carcinoma (327)

Figure 1. The expression of TCTN1 in thyroid cancer and nomal tissure by using Oncomine database.

may exist in thyroid cancer cell lines. Hence, we determined the expression of TCTN1 in three thyroid cancer cell lines (CAL-62,8305C and TT) and 293T cells. The results were listed in Fig. 1A. It can be seen from the results that TCTN1 expression in thyroid cancer cells was upregulated significant compared with that in $293 \mathrm{~T}$ cells. In order to explore the biological function of TCTN1 in the thyroid cancer; we used lentivirus-mediated shRNA to knockdown the endogenous TCTN1 gene expression in CAL62 and 8305C cells (undifferentiated carcinoma). After 7 days infection, we observed the GFP fluorescence light under the fluorescence microscope and found higher virus infection rate (Fig. 2A). Then we used real-time PCR to check the mRNA expression level of TCTN1, and the results showed that comparing with the control group, the mRNA amount of TCTN1 in the shTCTN1 group was largely decreased (Fig. 2B). Moreover, western blot analysis also confirmed that TCTN1 protein level in shTCTN1 group was significantly decreased comparing with that in shCon group in CAL62 and 8305C cells (Fig. 2C). These results showed that, lentivirus-mediated shRNA we designed could efficiently suppress the endogenous TCTN1 in thyroid cancer cells.

However, the current study is affected by several limitations, including the absence of a comparison between TCTN1 expression in tumor tissues and adjacent paired non-cancerous tissues, as well as that in normal thyroid cell lines vs. thyroid cancer cell lines. Thus, further experiments are required to clarify the endogenous expression of TCTN1 in thyroid cancer.

Downregulation of TCTN1 suppressed the cell proliferation in thyroid cancer cells. Using MTT assay, we went on to check the influence of TCTN1 gene silencing on thyroid cancer cell viability. We observed that after lentivirus infection, the cell proliferation rate of the TCTN1 knockdown group was obviously decreased compared with the control group in both CAL62 and 8305C cells (Fig. 3A). Furthermore, lots of colonies were observed in cells in the shCon group, but fewer colonies were formed in shTCTN1 group in CAL62 cells (Fig. 3B). Statistical analysis further demonstrated that the number of colony in shTCTN1 group was decreased by $92.03 \%$ (Fig. 3C, $\mathrm{P}<0.001$ ), suggesting that knockdown of TCTN1 significantly impaired the proliferation of thyroid cancer cells.

Downregulation of TCTN1 induced cell cycle arrest. We used flow cytometer with PI staining to detect the effect of TCTN1 silencing on cell cycle progression in CAL62 and 8305C cells (Fig. 4). The results (Fig. 4C and E) revealed that knockdown of TCTN1 induced a decrease in the percentage of cells in G0/G1 phase in CAL62 cells (from 50.86\% in shCon to $46.89 \%$ in shTCTN1 group, $\mathrm{P}<0.01$ ) and $8305 \mathrm{C}$ cells 
A

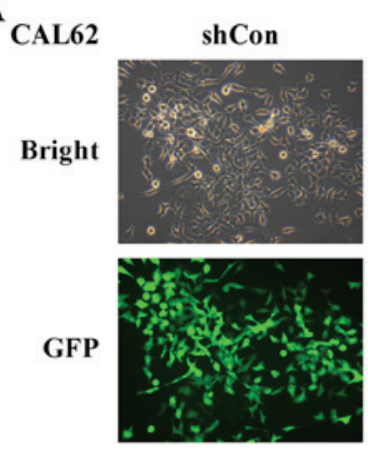

\begin{tabular}{|l|l|}
\hline 8305C & shCon \\
\hline Bright & \\
\hline & \\
\hline
\end{tabular}

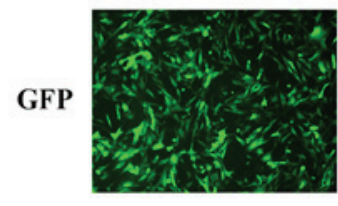

shTCTN1

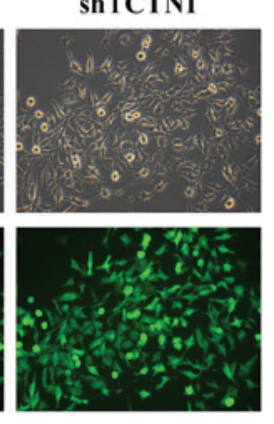

shTCTN1

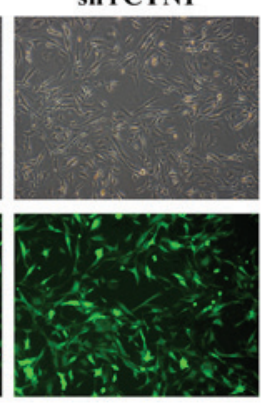

B
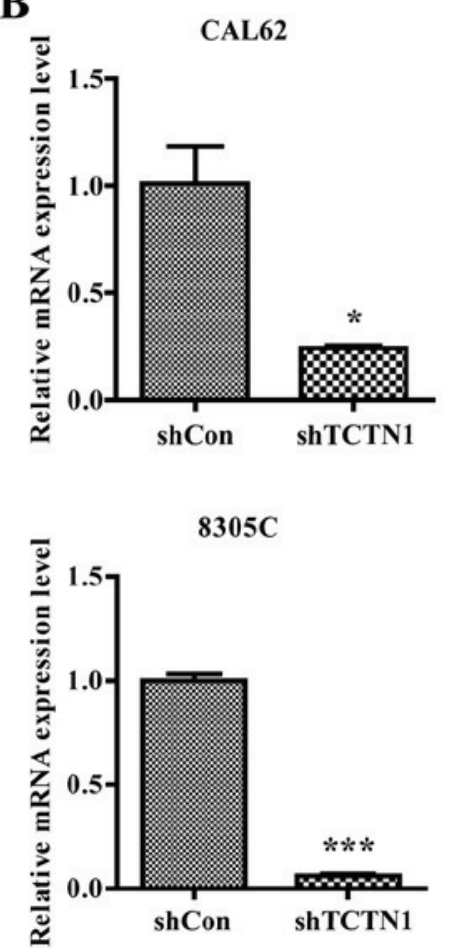

C
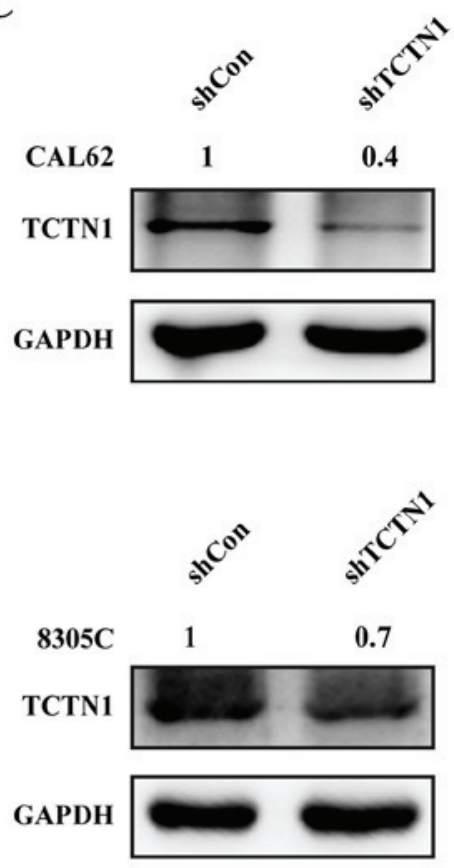

Figure 2. Knockdown of TCTN1 expression in thyroid cancer cells by shTCTN1. (A) Representative pictures of green fluorescent protein (GFP) expression recorded under a fluorescence microscope in CAL62 and 8305C cells. (B) qRT-PCR analysis of TCTN1 mRNA levels in CAL62 and 8305C cells following shCon or shTCTN1 infection. Data are expressed as mean \pm SD of 3 independent experiments. ${ }^{*} \mathrm{P}<0.05$, ${ }^{* * *} \mathrm{P}<0.001$ vs. shCon. (C) Western blot analysis of TCTN1 protein levels in CAL62 and 8305C cells following shCon or shTCTN1 infection. The number in this figure indicated the relative expression of TCTN1 relative to GAPDH. GAPDH was used as an internal control.

(from $73.36 \%$ in shCon to $61.63 \%$ in shTCTN1 group, $\mathrm{P}<0.01$ ). Notably, we observed a significant increase in percentage of cells in $\mathrm{S}$ phase in CAL62 cells and $8305 \mathrm{C}$ cells $(\mathrm{P}<0.001)$. All these results demonstrated that down regulation of TCTN1 could arrest cell cycle at S phase in CAL62 cells and 8305 cells. Furthermore, more cells were obviously accumulated in the sub-G1 phase representing apoptotic cells in CAL62 and 8305C cells (Fig. 4D and F, $\mathrm{P}<0.05$ ).

Downregulation of TCTN1 promoted cell apoptotic in thyroid cancer cells. To further confirm whether depletion of TCTN1 really promoted cell apoptosis, we used flow cytometer with Annexin V/ADD double staining to determine apoptosis in CAL62 and 8305C cells (Fig. 5A). After shTCTN1 infection, more cells were presented early (Annexin V+/7-AAD-, $\mathrm{P}<0.05$ ) and late apoptosis (Annexin $\mathrm{V}+/ 7-\mathrm{AAD}+, \mathrm{P}<0.001$ ) in both CAL62 and 8305C cells (Fig. 5B).

Downregulation of TCTN1 regulated cell apoptotic and cell cycle markers. Moreover, we detected the expression alterations of apoptotic and cell cycle regulatory molecules. As shown in Fig. 6A and B, the expression levels of cleaved-PARP and cleaved caspase-3 were obviously unregulated in both CAL62 and $8305 \mathrm{C}$ cells after TCTN1 knockdown. Additionally, downregulation of TCTN1 slightly suppressed Bcl-2 expression and elevated Bad expression in CAL62 cells, but didn't obviously affect the expression levels of Bcl-2 and Bad in $8305 \mathrm{C}$, which might be ascribed to different cell resource and culture condition. Furthermore, downregulation of TCTN1 decreased Cyclin B1 expression, and increased the expression of CDK2, Cyclin A1 and Cyclin D1 in both CAL62 and 8305C cells (Fig. 6C and D).

\section{Discussion}

The hedgehog pathway, initially discovered by two Nobel laureates Dr C. Nusslein-Volhard and Dr E. Wieschaus in Drosophila (15), is a major regulator for cell differentiation, tissue polarity and cell proliferation. Studies from many laboratories reveal activation of this pathway in a variety of human cancer, including basal cell carcinomas (BCCs), medulloblastomas, leukemia, gastrointestinal, lung, ovarian, breast and prostate cancers $(16,17)$. TCTN1, as a new discovered Hedgehog pathway bidirectional regulator, could activate/repress the hedgehog signal pathway during mouse embryogenesis (5). Based on these evidence, we speculated that TCTN1 might play a crucial role in thyroid cancer. Thus, in the present study, we evaluated the functional significance of TCTN1 in thyroid cancer and its potential as a therapeutic target. Lenvitirus-mediated shRNA was firstly designed to specifically targeted TCTN1 gene in thyroid cancer cell lines, CAL62 and 8305C cells. Loss-of-function analysis indicated that knockdown of TCTN1 significantly suppressed cell proliferation and colony formation in thyroid cancer cells. Our data were in accordance with previous report which studied the effects of TCTN1 knockdown in gastric cancer (10) and medulloblastoma (18). In addition, it should be pointed that the data obtained in the present study should be validated in future 
$\mathbf{A}$

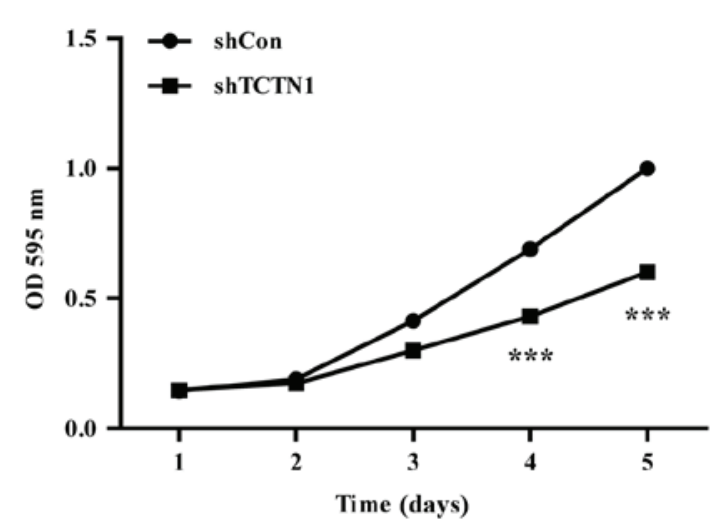

B

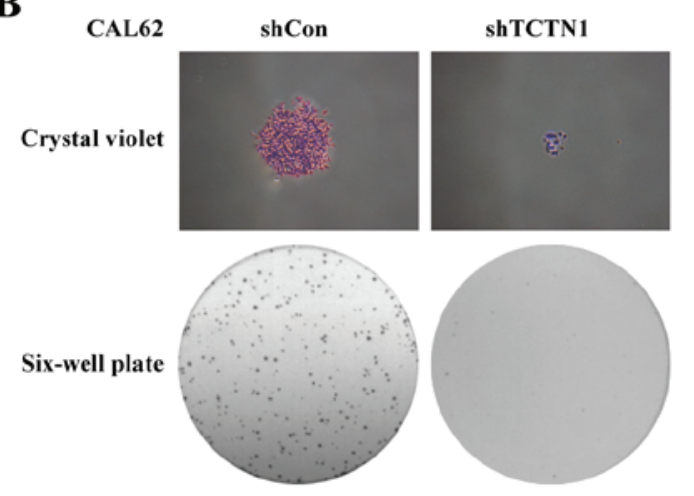

C
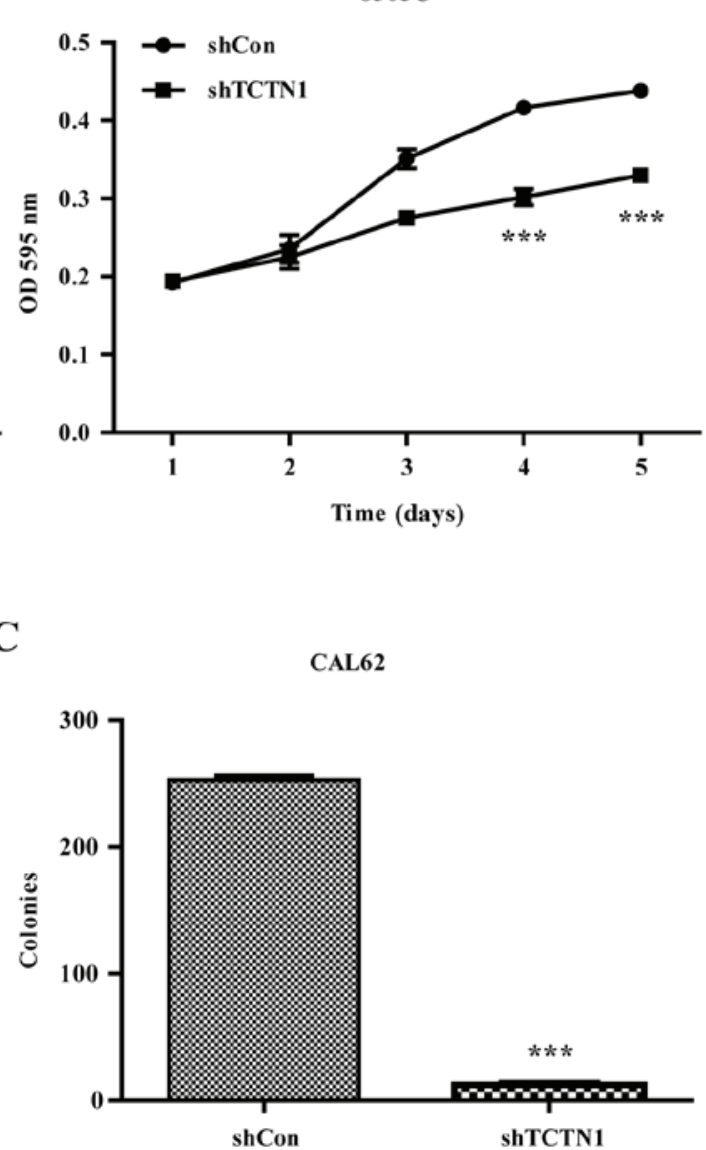

Figure 3. Knockdown of TCTN1 suppressed cell proliferation of thyroid cancer cells. (A) MTT assay was performed to analyze cell viability in CAL62 and 8305C cells following shCon or shTCTN1 infection. (B) Representative images of colonies formed in CAL62 cells after shCon or shTCTN1 infection recorded under a microscope. (C) Statistical analysis of colonies in CAL62 cells after shCon or shTCTN1 infection. Data are expressed as mean \pm SD of 3 independent experiments. ${ }^{* * *} \mathrm{P}<0.001$ vs. shCon.

A

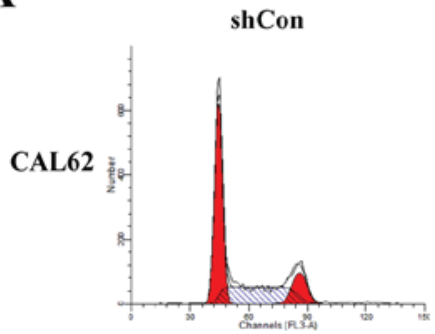

C

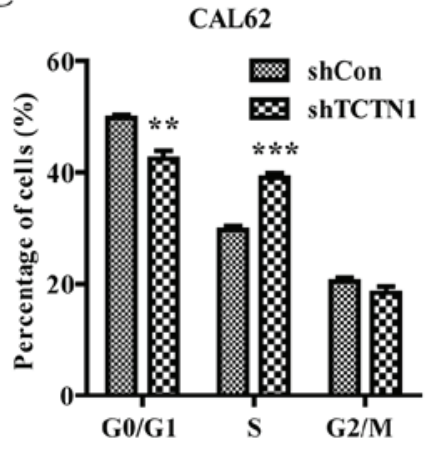

$\operatorname{shTCTN1}$

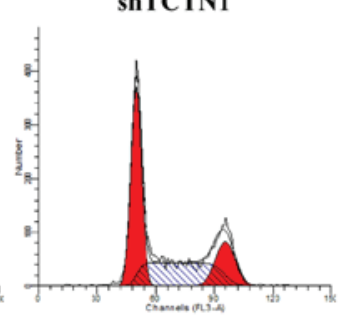

D
B $8305 C$

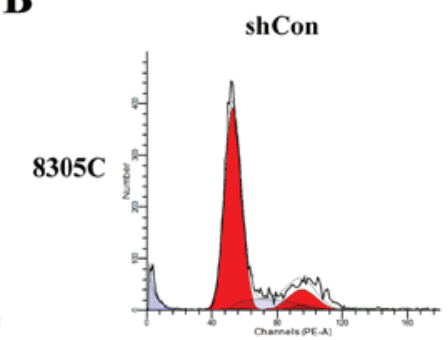

$\mathbf{E}$

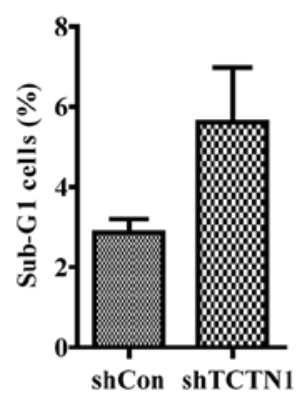

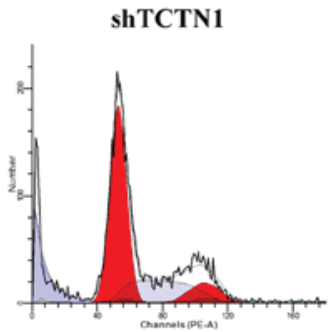

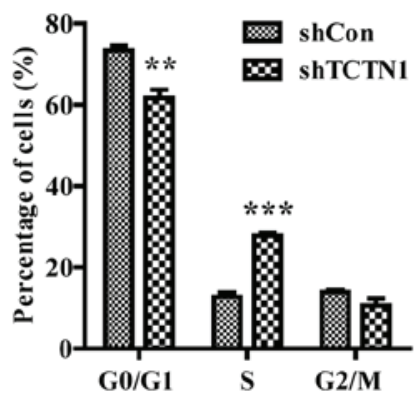

$\mathbf{F}$

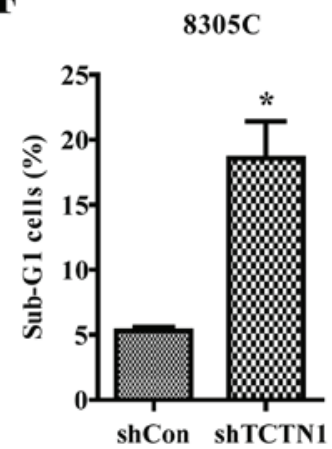

Figure 4. Knockdown of TCTN1 induced cell cycle arrest at S phase in thyroid cancer cells. (A and B) Flow cytometry with PI staining was used to analyze the cell cycle distribution of CAL62 and 8305C cells following shCon or shTCTN1 infection. Statistical analysis of cell percentage in G0/G1, S, G2/M and sub-G1 phases in CAL62 (C and D) and 8305C (E and F) cells following shCon or shTCTN1 infection; Data are expressed as mean \pm SD of 3 independent experiments. ${ }^{*} \mathrm{P}<0.05,{ }^{* *} \mathrm{P}<0.01,{ }^{* * *} \mathrm{P}<0.001$ vs. shCon. 


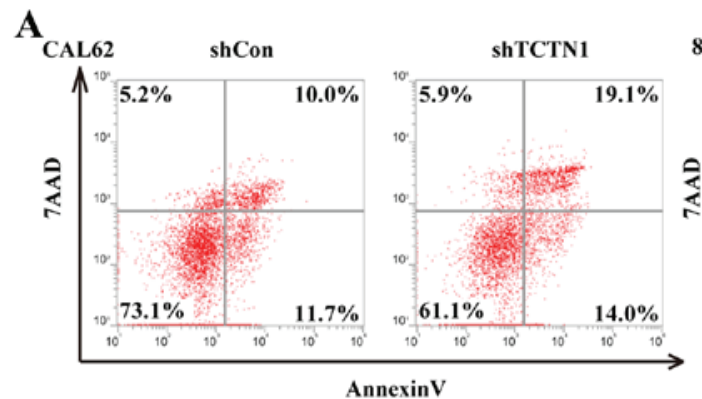

B

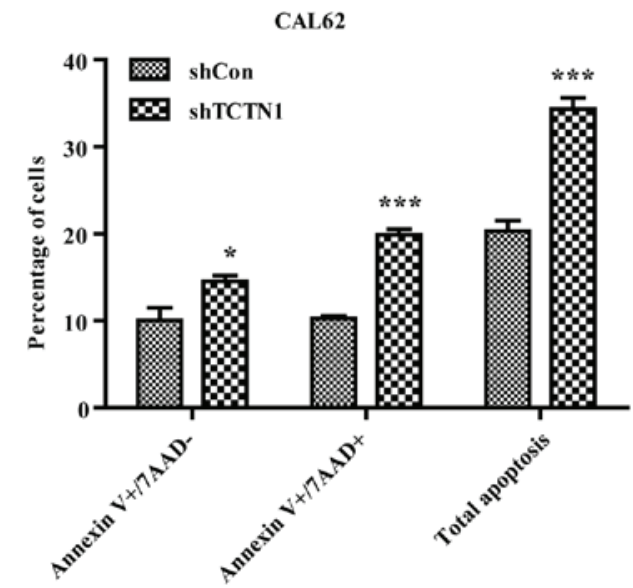

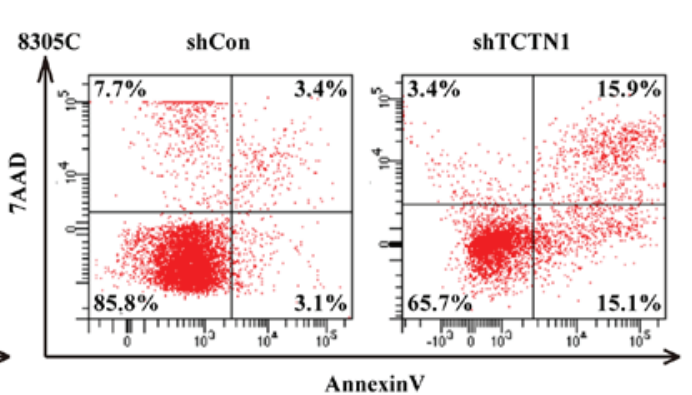

8305C

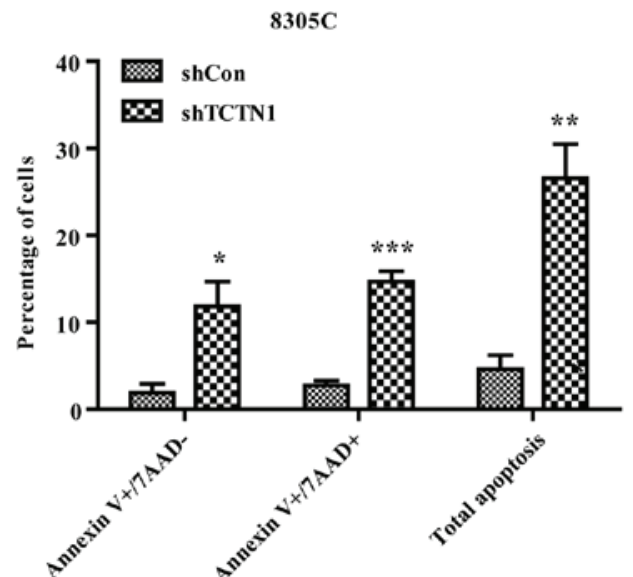

Figure 5. Knockdown of TCTN1 induced cell apoptosis in thyroid cancer cells. (A) Flow cytometry with Annexin V-APC/7-AAD double staining was used to analyze cell apoptosis in CAL62 and 8305C cells following shCon or shTCTN1 infection. (B) Statistical analysis of apoptotic cell proportion, including early apoptosis (Annexin V +/7-AAD-) and late apoptosis (Annexin V +/7-AAD+) in CAL62 and 8305C cells following shCon or shTCTN1 infection. Data are expressed as mean \pm SD of 3 independent experiments. ${ }^{*} \mathrm{P}<0.05,{ }^{* *} \mathrm{P}<0.01,{ }^{* * *} \mathrm{P}<0.001$ vs. shCon.

$\mathbf{A}$

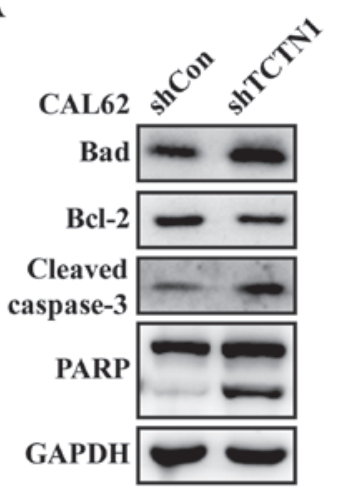

C

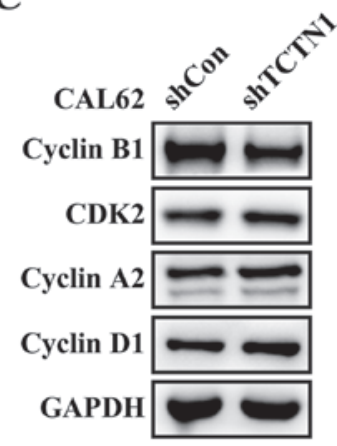

B

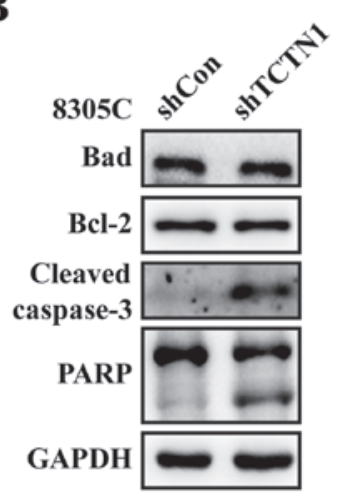

D

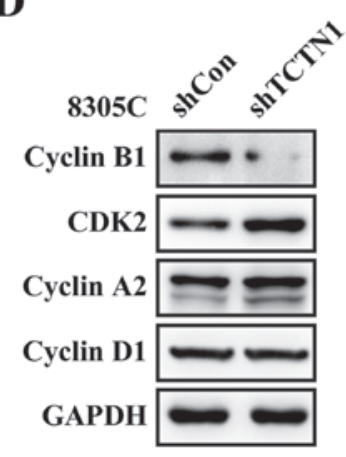

Figure 6. Knockdown of TCTN1 induced alteration in cell cycle and apoptotic regulator expression in thyroid cancer cells. Expression levels of Bad, Bcl-2, caspase-3 and PARP were analyzed with Western blotting in CAL62 (A) and 8305C (B) cells after infected with shCon or shTCTN1. Expression levels of Cyclin B1, CDK2, Cyclin A2 and Cyclin D1 were determined in CAL62 (C) and 8305C (D) cells after infected with shCon or shTCTN1 using western blotting. GAPDH was used as an internal control. 
studies using other kind of thyroid cancer cell lines, including papillary carcinoma follicular carcinoma and medullary carcinoma.

Furthermore, we found TCTN1 knockdown could arrest cell cycle at $\mathrm{S}$ phase in CAL62 cells and 8305 cells. Consistent with $\mathrm{S}$ phase arrest, the expression of cyclin $\mathrm{A} 2$ and CDK2, which are known to promote $S$ phase entry in mammals $(19,20)$, significantly increased following TCTN1 knockdown. Instead, we observed a dramatic reduction in cyclin B1 levels. Cyclin B1 is a key regulator of cell cycle progression from the $\mathrm{S}$ phases to the $\mathrm{G} 2 / \mathrm{M}$ phase and its downregulation therefore results in $\mathrm{S}$ phase arrest (21). Similarly, cyclin D1 is involved in normal cell cycle progression. In addition, we observed there was a significant early and late apoptosis in CAL62 and 8305C cells after TCTN1 knockdown, which further demonstrated TCTN1 played a positive role in thyroid cancer cell growth and proliferation. To illuminate the molecular mechanisms by which TCTN1 affected thyroid cancer apoptosis, we detected some molecules associated with cell survival in CAL62 cells after TCTN1 knockdown. The anti-apoptotic protein Bcl-2 plays a pivotal role in regulating cell apoptosis under many adverse conditions, while pro-apoptotic Bad is essential for inducing apoptosis (22). In our results, reduced Bcl-2 and increased Bad expression suggested silencing TCTN1 might serve as a promoter of cell apoptosis. Notably, a significant proteolytic cleavage of PARP and caspase-3 was detected, also indicating a pro-apoptotic role for TCTN1 knockdown. These results are supported by the previous study that TCTN1 silencing could promote cell apoptosis in pancreatic cancer (12). Collectively, inhibition of cell proliferation in thyroid cancer induced by TCTN1 knockdown might be closely associated with cell cycle arrest and apoptosis in thyroid cancer cells.

In conclusion, the present study demonstrates that TCTN1 plays a crucial role in promoting thyroid cancer cell growth and proliferation in vitro. The TCTN1-targetd therapy using RNA interference potential application may provide a novel therapy for thyroid cancer. Furthermore, investigations on TCTN1 function in vivo are still necessary for preclinical and clinical studies.

\section{Acknowledgements}

The present study was supported by Shanghai Science and Technology Commission Funded Project (grant no. 14411964100).

\section{References}

1. Liebner DA and Shah MH: Thyroid cancer: Pathogenesis and targeted therapy. Ther Adv Endocrinol Metab 2: 173-195, 2011.

2. Pacifico F and Leonardi A: Role of NF-kappaB in thyroid cancer. Mol Cell Endocrinol 321: 29-35, 2010.

3. Sipos JA and Mazzaferri EL: Thyroid cancer epidemiology and prognostic variables. Clin Oncol (R Coll Radiol) 22: 395-404, 2010 .
4. Garcia-Gonzalo FR, Corbit KC, Sirerol-Piquer MS, Ramaswami G, Otto EA, Noriega TR, Seol AD, Robinson JF, Bennett CL, Josifova DJ, et al: A transition zone complex regulates mammalian ciliogenesis and ciliary membrane composition. Nat Genet 43: 776-784, 2011.

5. Reiter JF and Skarnes WC: Tectonic, a novel regulator of the Hedgehog pathway required for both activation and inhibition. Genes Dev 20: 22-27, 2006.

6. Parisi M and Glass I: Joubert syndrome and related disorders. In: Gene Reviews (R). Pagon RA, Adam MP, Ardinger HH, et al (eds.): Seattle (WA), 1993.

7. Alazami AM, Alshammari MJ, Salih MA, Alzahrani F, Hijazi H, Seidahmed MZ, Abu Safieh L, Aldosary M, Khan AO, Alkuraya FS, et al: Molecular characterization of Joubert syndrome in Saudi Arabia. Hum Mutat 33: 1423-1428, 2012.

8. Huppke P, Wegener E, Bohrer-Rabel H, Bolz HJ, Zoll B, Gärtner $\mathbf{J}$ and Bergmann C: Tectonic gene mutations in patients with Joubert syndrome. Eur J Hum Genet 23: 616-620, 2015.

9. Li J, Wang H, Hang C, Fan Y, Ma C and Pan Y: Lentivirus-mediated knockdown of TCTN1 inhibits glioma cell proliferation. Appl Biochem Biotechnol 176: 13-21, 2015.

10. Wang X, Yu Q, Zhang Y, Ling Z and Yu P: Tectonic 1 accelerates gastric cancer cell proliferation and cell cycle progression in vitro. Mol Med Rep 12: 5897-5902, 2015.

11. Wang Z, Gao YI, Liu Y, Chen J, Wang J, Gan S, Xu D and Cui X: Tectonic-1 contributes to the growth and migration of prostate cancer cells in vitro. Int J Mol Med 36: 931-938, 2015.

12. Zhao S, Chen X, Wan M, Jiang X, Li C, Cui Y and Kang P: Tectonic 1 is a key regulator of cell proliferation in pancreatic cancer. Cancer Biother Radiopharm 31: 7-13, 2016.

13. Zhang J, Liang Q, Lei Y, Yao M, Li L, Gao X, Feng J, Zhang Y, Gao H, Liu DX, et al: SOX4 induces epithelial-mesenchymal transition and contributes to breast cancer progression. Cancer Res 72: 4597-4608, 2012.

14. Shen G, Jia H, Tai Q, Li Y and Chen D: miR-106b downregulates adenomatous polyposis coli and promotes cell proliferation in human hepatocellular carcinoma. Carcinogenesis 34: 211-219, 2013.

15. Nusslein-Volhard C, Wieschaus E. Mutations affecting segment number and polarity in Drosophila. Nature 287: 795-801, 1980.

16. Shaw G, Price AM, Ktori E, Bisson I, Purkis PE, McFaul S, Oliver RT and Prowse DM: Hedgehog signalling in androgen independent prostate cancer. Eur Urol 54: 1333-1343, 2008.

17. Hui M, Cazet A, Nair R, Watkins DN, O'Toole SA and Swarbrick A: The Hedgehog signalling pathway in breast development, carcinogenesis and cancer therapy. Breast Cancer Res 15: 203, 2013.

18. Jing J, Wang C, Liang Q, Zhao Y, Zhao Q, Wang S and Ma J: Lentivirus-mediated knockdown of tectonic family member 1 inhibits medulloblastoma cell proliferation. Int J Clin Exp Med 8: 13127-13135, 2015.

19. Yin H, Subrata S, Yang W and Yang G: Overexpression of cyclin A leads to S-phase arrested apoptosis. Hua Xi Yi Ke Da Xue Xue Bao 32: 188-190, 2001 (In Chinese).

20. Ding H, Han C, Guo D, Wang D, Chen CS and D'Ambrosio SM: OSU03012 activates Erk1/2 and Cdks leading to the accumulation of cells in the S-phase and apoptosis. Int J Cancer 123: 2923-2930, 2008.

21. Xu X, Zhang H, Zhang Q, Huang Y, Dong J, Liang Y, Liu HJ and Tong D: Porcine epidemic diarrhea virus $\mathrm{N}$ protein prolongs $\mathrm{S}$-phase cell cycle, induces endoplasmic reticulum stress, and up-regulates interleukin-8 expression. Vet Microbiol 164: 212-221, 2013.

22. Si Y, Zhang Y, Han L, Chen L, Xu Y, Sun F, Ji M, Yang J and Bao H: Dexmedetomidine acts via the JAK2/STAT3 pathway to attenuate isoflurane-induced neurocognitive deficits in senile mice. PloS One 11: e0164763, 2016. 